\title{
Physics with large extra dimensions
}

\section{Ignatios Antoniadis*†}

CERN, Theory Division, CH-1211, Geneva 23, Switzerland

E-mail: Ignatios.Antoniadis@cern.ch

\begin{abstract}
The recent understanding of string theory opens the possibility that the string scale can be as low as a few $\mathrm{TeV}$. The apparent weakness of gravitational interactions can then be accounted by the existence of large internal dimensions, in the submillimeter region. Furthermore, our world must be confined to live on a brane transverse to these large dimensions, with which it interacts only gravitationally. In my lecture, I describe briefly this scenario which gives a new theoretical framework for solving the gauge hierarchy problem and the unification of all interactions. I also discuss its main properties and implications for observations at both future particle colliders, and in non-accelerator gravity experiments. Such effects are for instance the production of Kaluza-Klein resonances, graviton emission in the bulk of extra dimensions, and a radical change of gravitational forces in the submillimeter range.
\end{abstract}

\section{Introduction}

In all physical theories, the number of dimensions is a free parameter fixed to three by observation, with one exception: string theory, which predicts the existence of six new spatial dimensions. This is the only known theory today that unifies the two great discoveries of 20th century: quantum mechanics, describing the behavior of elementary particles, and Einstein's General Relativity, describing gravitational phenomena in our Universe.

String theory replaces all elementary point-particles that form matter and its interactions with a single extended object of vanishing width: a tiny string. Thus, every known elementary particle, such as the electron, quark, photon or neutrino, corresponds to a particular vibration mode of the string (see Fig. 1).

The diversity of these particles is due to the different properties of the corresponding string vibrations.

How can it be tested? If our universe has really six additional dimensions, we should observe new phenomena related to the existence of these dimensions. Why nobody has

${ }^{*}$ Speaker.

${ }^{\dagger}$ On leave from CPHT (UMR 7644 of CNRS), Ecole Polytechnique, F-91128 Palaiseau. 


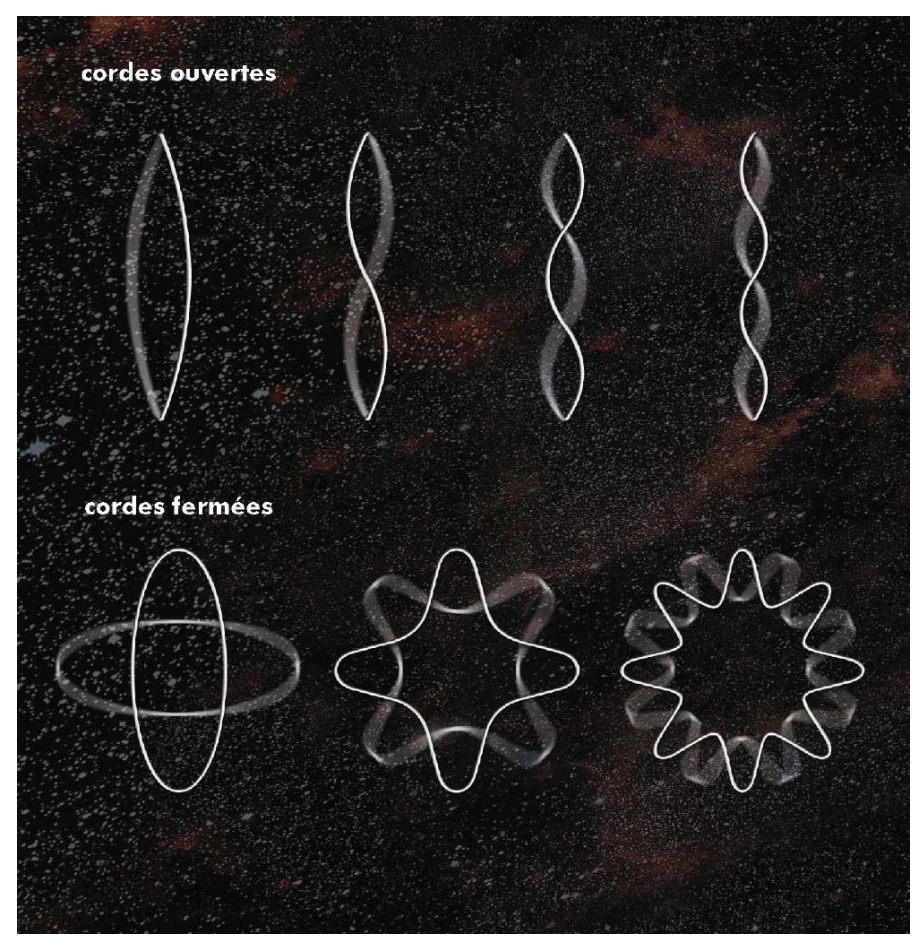

Figure 1: In string theory, the elementary constituent of matter is a miniscule string, having vanishing width but finite size. It can be open with free ends (upper part), or closed (lower part). Its vibration modes, like the ones shown above in two dimensions, correspond to various elementary particles.

detected them until now? String theorists had an answer for a long time: because the size of the new dimensions is very small, in contrast to the size of the other three that we know, which is infinitely large.

An infinite and narrow cylinder for example is a two-dimensional space, with one dimension forming a very small cycle: one can move infinitely far away along the axis, while one returns back at the same point when moving along the orthogonal direction (see Fig. 2).

If one of the three known dimensions of space was small, say of millimeter size, we would be flat and, while we could move freely towards left or right, forward or backward, it would be impossible to do more than a few millimeters up or down where space ends.

For a long time, string physicists thought that the six extra dimensions were extremely small, having the smallest possible size of physics, associated to the Planck length $\sim 10^{-35}$ meters. In fact, strings were introduced to describe gravitation whose strength becomes important and comparable to the strength of the other three fundamental interactions (electromagnetic, nuclear strong and weak) at very short distances, of the order of the Planck length. It was then natural to assume that the size of the extra dimensions should be of the same order. In this case, the manifestation of new phenomena associated to the extra dimensions are by far out of experimental reach, at least in particle accelerators. Indeed, the Large Hadron Collider (LHC) which is the biggest accelerator under construction at 
CERN will explore short distances, only up to $10^{-19}$ meters.

The situation changed drastically recently. Dur-

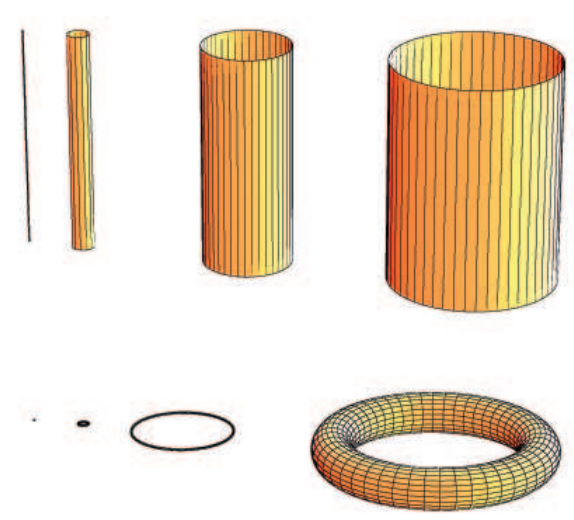

Figure 2: Possible forms of small extra dimensions of space. Far away they are unobservable, but at distances comparable to their size we start feeling their existence and exploring their shapes. ing the last three years, more and more theorists examine the possibility that the new dimensions of string theory may be much larger than we thought in the past [1, 2]. These ideas lead in particular to experimental tests of string theory that can be performed at TEVATRON and LHC, or at future colliders.

\section{The universe as a braneworld}

A particularly attractive scenario is when the string scale is in the $\mathrm{TeV}$ region, which stabilizes the mass hierarchy problem without need of supersymmetry [2]. A possible realization of this idea without experimental conflict is in models possessing large extra dimensions along which only gravity propagates: gravity appears to us very weak at macroscopic scales because its intensity is spread in the "hidden" extra dimensions. On the other hand, at $\mathrm{TeV}$ energies, it becomes comparable in strength with the other interactions, i.e. $10^{32}$ times stronger than what we believed in the past. In order to increase the gravitational force without contradicting present observations, one has to introduce at least two such extra dimensions of size that can be as large as a fraction of a millimeter. At these distances, gravity should start deviate from Newton's law, which may be possible to explore in laboratory experiments [3] (see Fig. 3).

A convenient perturbative framework realizing this idea is one of the five string theories, called type I, that contains simultaneously closed and open strings [2. Our universe should be localized on a hypersurface, i.e. a membrane extended in $p$ spatial dimensions with $p<7$, called $p$-brane (see Fig. 目). Closed strings describe gravity and propagate in all nine dimensions of space: in those extended along the $p$-brane, as well as in the transverse ones. On the contrary, the endpoints of open strings describing the other (gauge) interactions are confined on the $p$-brane.

Obviously, our $p$-braneworld must have at least the three known dimensions of space. But it may contain more as opposed to the transverse dimensions that interact with us only gravitationally, the "longitudinal" to the brane extra dimensions can be "seen" by the light at sufficiently high energies, giving rise to the production of massive Kaluza-Klein particles in accelerators (see Fig. 5) [四.

On the other hand, the existence of the extra large (sub)millimeter dimensions, transverse to our $p$-brane universe, guarantee that gravitational interactions appear to us very weak at macroscopic distances, larger that a millimeter. The size of these transverse dimensions varies from a fraction of millimeter (in the case of two) to a Fermi $\left(10^{-14}\right.$ meters, in the case of six). Their characteristic signal in particle colliders is graviton emission into 


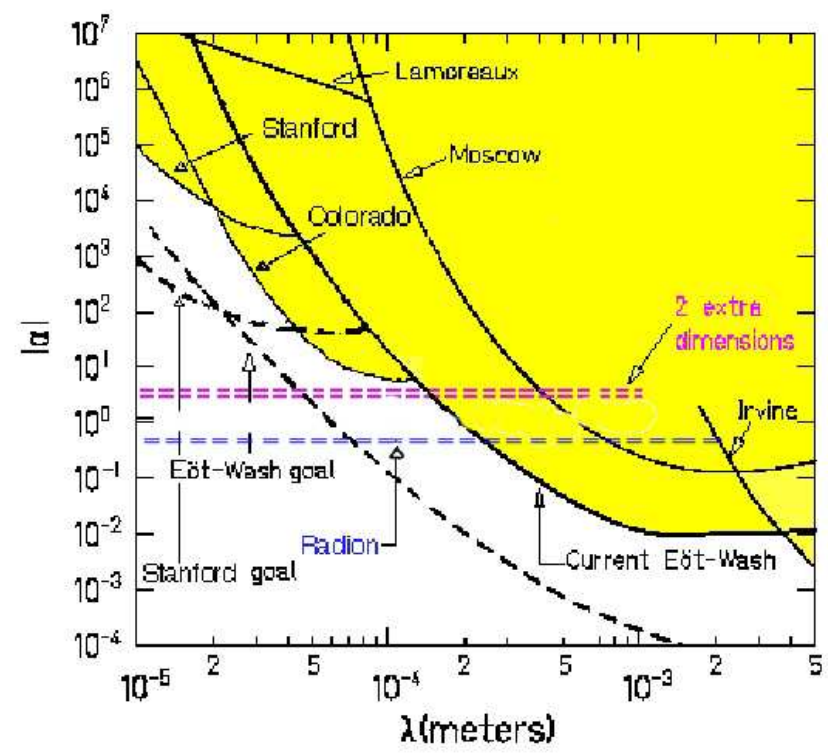

Figure 3: Present limits on non-Newtonian forces at short distances (yellow regions), as a function of their range $\lambda$ (horizontal axis) and their strength relative to gravity $\alpha$ (vertical axis). The limits are compared to new forces mediated by the graviton in the case of two large extra dimensions, and by the radion.

the bulk, leading to missing energy that escapes detection [2, 2] (see Fig. 6). The limits on the size $R_{\perp}$ of transverse dimensions are summarized in Table 1 .

\begin{tabular}{|c|c|c|c|}
\hline & & & \\
Experiment & $R_{\perp}(n=2)$ & $R_{\perp}(n=4)$ & $R_{\perp}(n=6)$ \\
\hline \hline \multicolumn{4}{|c|}{ Collider bounds } \\
\hline LEP 2 & $4.8 \times 10^{-1}$ & $1.9 \times 10^{-8}$ & $6.8 \times 10^{-11}$ \\
\hline Tevatron & $5.5 \times 10^{-1}$ & $1.4 \times 10^{-8}$ & $4.1 \times 10^{-11}$ \\
\hline LHC & $4.5 \times 10^{-3}$ & $5.6 \times 10^{-10}$ & $2.7 \times 10^{-12}$ \\
\hline NLC & $1.2 \times 10^{-2}$ & $1.2 \times 10^{-9}$ & $6.5 \times 10^{-12}$ \\
\hline \hline \multicolumn{4}{|c|}{ Present non-collider bounds } \\
\hline SN1987A & $3 \times 10^{-4}$ & $1 \times 10^{-8}$ & $6 \times 10^{-10}$ \\
\hline COMPTEL & $5 \times 10^{-5}$ & - & - \\
\hline
\end{tabular}

Table 1: Limits on $R_{\perp}$ in mm from missing-energy processes, as well as from astrophysics and cosmology.

\section{D-brane Standard Model}

One of the main questions with such a low string scale is to understand the observed values of the low energy gauge couplings. One possibility is to have the three gauge group factors 


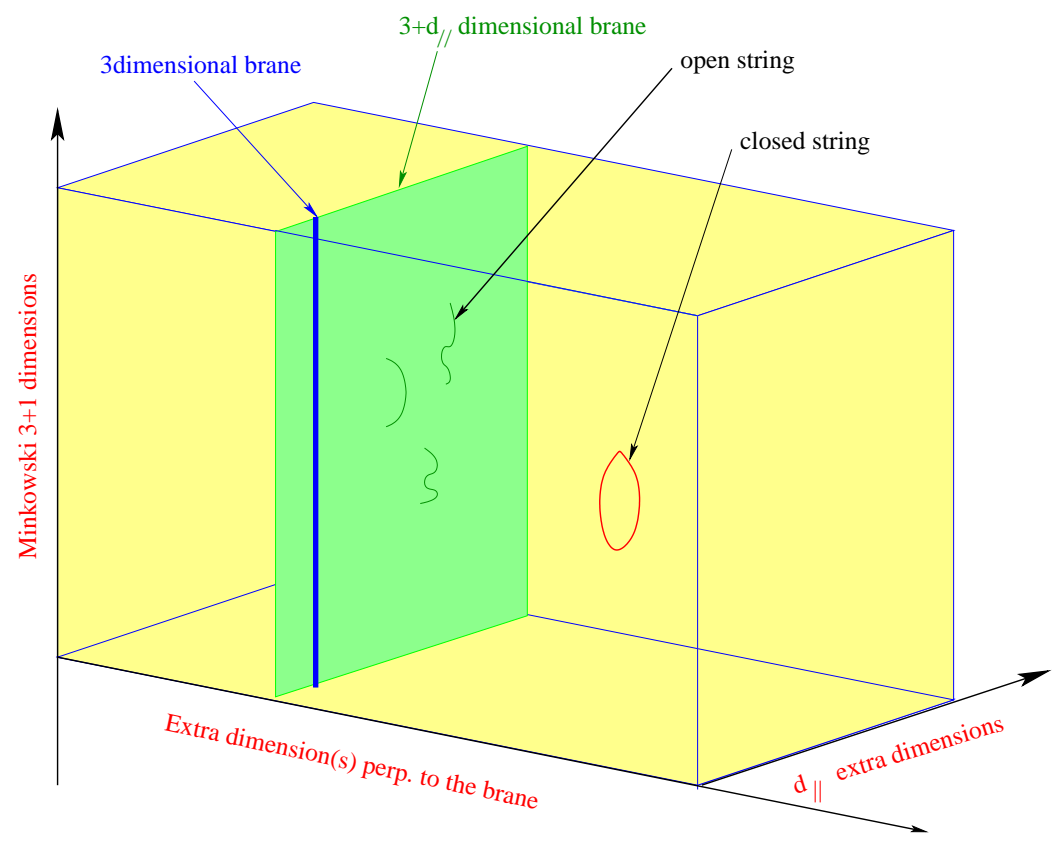

Figure 4: In the type I string framework, our Universe contains, besides the three known spatial dimensions (denoted by a single blue line), some extra dimensions $\left(d_{\|}=p-3\right)$ parallel to our world $p$-brane (green plane) along which the light described by open strings propagates, as well as some transverse dimensions (yellow space) where only gravity described by closed strings can propagate. The longitudinal extra dimensions have the string size of the order of $10^{-18}$ meters, while the size of the transverse dimensions varies in the range of $10^{-14}$ meters to a fraction of a millimeter.

of the Standard Model (SM) arising from different collections of coinciding branes. This is unattractive since the three gauge couplings correspond in this case to different arbitrary parameters of the model. A second possibility is to maintain unification by imposing all the SM gauge bosons to arise from the same collection of D-branes. The large difference in the actual values of gauge couplings could then be explained either by introducing power-law running from a few $\mathrm{TeV}$ to the weak scale [6], or by an effective logarithmic evolution in the transverse space in the special case of two large dimensions [7]. However, no satisfactory model built along these lines has so far been presented.

Here, we will discuss a third possibility [8, which is alternative to unification but nevertheless maintains the prediction of the weak angle at low energies. Specifically, we consider the strong and electroweak interactions to arise from two different collections of coinciding branes, leading to two different gauge couplings, [9]. Assuming that the low energy spectrum of the (non-supersymmetric) SM can be derived by a type I string vacuum, the normalization of the hypercharge is determined in terms of the two gauge couplings and leads naturally to the right value of $\sin ^{2} \theta_{W}$ for a string scale of the order of a few $\mathrm{TeV}$. The electroweak gauge symmetry is broken by the vacuum expectation values of two Higgs doublets, which are both necessary in the present context to give masses to all quarks and leptons. 


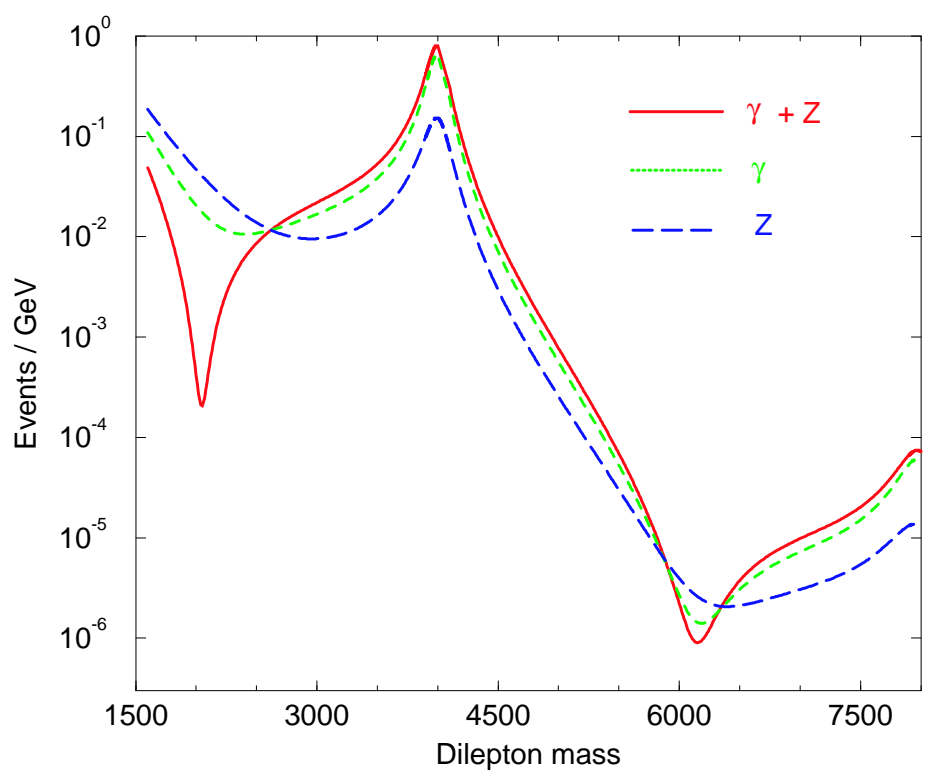

Figure 5: If there is an extra dimension of size $10^{-18}$ meters, felt by the electroweak interactions, LHC should produce the first Kaluza-Klein states of the photon and of the $Z$ boson. We can then detect the electron-positron pairs produced by the disintegration of these states. The number of the expected events is computed as a function of the energy of the pair in GeV. From highest to lowest: excitation of photon $+\mathrm{Z}$, photon and $\mathrm{Z}$ boson.

Another issue of this class of models with $\mathrm{TeV}$ string scale is to understand proton stability. In the model presented here, this is achieved by the conservation of the baryon number which turns out to be a perturbatively exact global symmetry, remnant of an anomalous $U(1)$ gauge symmetry broken by the Green-Schwarz mechanism. Specifically, the anomaly is canceled by shifting a corresponding axion field that gives mass to the $U(1)$ gauge boson. Moreover, the two extra $U(1)$ gauge groups are anomalous and the associated gauge bosons become massive with masses of the order of the string scale. Their couplings to the standard model fields up to dimension five are fixed by charges and anomalies.

\subsection{Hypercharge embedding and the weak angle}

The gauge group closest to the Standard Model one can hope to derive from type I string theory in the above context is $U(3) \times U(2) \times U(1)$. The first factor arises from three coincident "color" D-branes. An open string with one end on them is a triplet under $S U(3)$ and carries the same $U(1)$ charge for all three components. Thus, the $U(1)$ factor of $U(3)$ has to be identified with gauged baryon number. Similarly, $U(2)$ arises from two coincident "weak" D-branes and the corresponding abelian factor is identified with gauged weak-doublet number. A priori, one might expect that $U(3) \times U(2)$ would be the minimal choice. However it turns out that one cannot give masses to both up and down quarks in that case. Therefore, at least one additional $U(1)$ factor corresponding to an extra " $U(1)$ " D-brane is necessary in order to accommodate the Standard Model. In principle this $U(1)$ 


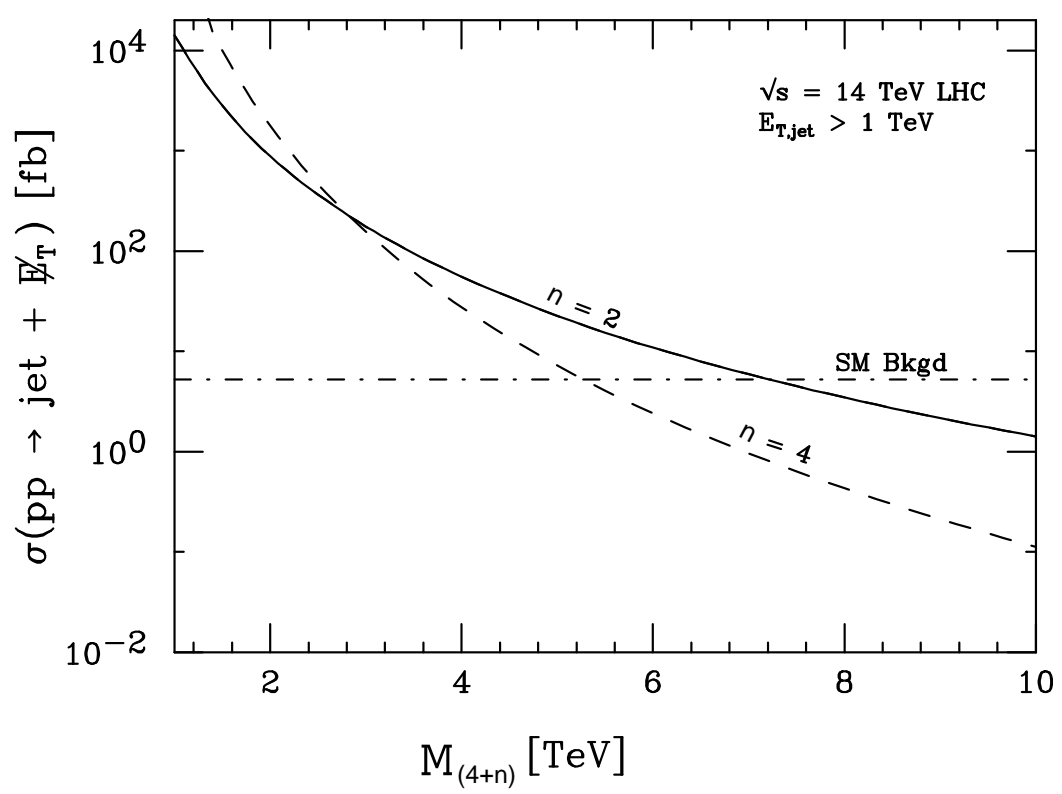

Figure 6: Missing energy due to graviton emission in the LHC experiment, as a function of the fundamental scale $M_{(4+n)}$ of quantum gravity that propagates in $n$ large transverse dimensions. It is produced together with a hadronic jet that one detects in the collision of the two proton beams. The figure shows the expected cross-section for $n=2$ and $n=4$ extra dimensions, together with the background (horizontal dotted-dashed line) coming from other known sources.

brane can be chosen to be independent of the other two collections with its own gauge coupling. To improve the predictability of the model, here we choose to put it on top of either the color or the weak D-branes. In either case, the model has two independent gauge couplings $g_{3}$ and $g_{2}$ corresponding, respectively, to the gauge groups $U(3)$ and $U(2)$. The $U(1)$ gauge coupling $g_{1}$ is equal to either $g_{3}$ or $g_{2}$.

Let us denote by $Q_{3}, Q_{2}$ and $Q_{1}$ the three $U(1)$ charges of $U(3) \times U(2) \times U(1)$, in a self explanatory notation. Under $S U(3) \times S U(2) \times U(1)_{3} \times U(1)_{2} \times U(1)_{1}$, the members of a family of quarks and leptons have the following quantum numbers:

$$
\begin{aligned}
& Q(\mathbf{3}, \mathbf{2} ; 1, w, 0)_{1 / 6} \\
& u^{c}(\overline{\mathbf{3}}, \mathbf{1} ;-1,0, x)_{-2 / 3} \\
& d^{c}(\overline{\mathbf{3}}, \mathbf{1} ;-1,0, y)_{1 / 3} \\
& L(\mathbf{1}, \mathbf{2} ; 0,1, z)_{-1 / 2} \\
& l^{c}(\mathbf{1}, \mathbf{1} ; 0,0,1)_{1}
\end{aligned}
$$

Here, we normalize all $U(N)$ generators according toTr $T^{a} T^{b}=\delta^{a b} / 2$, and measure the corresponding $U(1)_{N}$ charges with respect to the coupling $g_{N} / \sqrt{2 N}$, with $g_{N}$ the $S U(N)$ coupling constant. Thus, the fundamental representation of $S U(N)$ has $U(1)_{N}$ charge unity. The values of the $U(1)$ charges $x, y, z, w$ will be fixed below so that they lead to the right hypercharges, shown for completeness as subscripts.

The quark doublet $Q$ corresponds necessarily to a massless excitation of an open string with its two ends on the two different collections of branes. The $Q_{2}$ charge $w$ can be either 
+1 or -1 depending on whether $Q$ transforms as a $\mathbf{2}$ or a $\overline{\mathbf{2}}$ under $U(2)$. The antiquark $u^{c}$ corresponds to fluctuations of an open string with one end on the color branes and the other on the $U(1)$ brane for $x= \pm 1$, or on other branes in the bulk for $x=0$. Ditto for $d^{c}$. Similarly, the lepton doublet $L$ arises from an open string with one end on the weak branes and the other on the $U(1)$ brane for $z= \pm 1$, or in the bulk for $z=0$. Finally, $l^{c}$ corresponds necessarily to an open string with one end on the $U(1)$ brane and the other in the bulk.

The weak hypercharge $Y$ is a linear combination of the three $U(1)$ 's [10]:

$$
Y=c_{1} Q_{1}+c_{2} Q_{2}+c_{3} Q_{3} .
$$

$c_{1}=1$ is fixed by the charges of $l^{c}$ in eq. (3.1), while for the remaining two coefficients and the unknown charges $x, y, z, w$, we obtain four possibilities:

$$
\begin{gathered}
c_{2}=\mp \frac{1}{2}, c_{3}=-\frac{1}{3} ; x=-1, y=0, z=0 /-1, w=\mp 1 \\
c_{2}=\mp \frac{1}{2}, c_{3}=\frac{2}{3} ; x=0, y=1, z=0 /-1, w=\mp 1
\end{gathered}
$$

To compute the weak angle $\sin ^{2} \theta_{W}$, we use eq. (3.2) to find:

$$
\sin ^{2} \theta_{W} \equiv \frac{g_{Y}^{2}}{g_{2}^{2}+g_{Y}^{2}}=\frac{1}{1+4 c_{2}^{2}+2 g_{2}^{2} / g_{1}^{2}+6 c_{3}^{2} g_{2}^{2} / g_{3}^{2}}
$$

with $g_{1}=g_{2}$ or $g_{1}=g_{3}$ at the string scale.

We now show that the above prediction agrees with the experimental value for $\sin ^{2} \theta_{W}$ for a string scale in the region of a few $\mathrm{TeV}$. For this comparison, we use the evolution of gauge couplings from the weak scale $M_{Z}$ as determined by the one-loop beta-functions of the SM with three families of quarks and leptons and one Higgs doublet. In order to compare the theoretical relations for $g_{1}=g_{2}$ and $g_{1}=g_{3}$ with the experimental value of $\sin ^{2} \theta_{W}$ at $M_{s}$, we plot in Fig. 7 the corresponding curves as functions of $M_{s}$. The solid line is the experimental curve. The dashed line is the plot of the function (3.4) for $g_{1}=g_{2}$ with $c_{3}=-1 / 3$ while the dotted-dashed line corresponds to $g_{1}=g_{3}$ with $c_{3}=2 / 3$. The other two possibilities are not shown because they lead to a value of $M_{s}$ which is too high to protect the hierarchy. Thus, the second case, where the $U(1)$ brane is on top of the color branes, is compatible with low energy data for $M_{s} \sim 6-8 \mathrm{TeV}$ and $g_{s} \simeq 0.9$. This selects the last two possibilities of charge assignments in Eq. (3.3).

From the general solution (3.3) and the requirement that the Higgs doublet has hypercharge $1 / 2$, one finds the following possible assignments:

$$
c_{2}=\mp \frac{1}{2}: \quad H \quad(\mathbf{1}, \mathbf{2} ; 0, \pm 1,1)_{1 / 2} \quad H^{\prime} \quad(\mathbf{1}, \mathbf{2} ; 0, \mp 1,0)_{1 / 2}
$$

It is straightforward to check that the allowed (trilinear) Yukawa terms are:

$$
\begin{gathered}
c_{2}=-\frac{1}{2}: H^{\prime} Q u^{c}, H^{\dagger} L l^{c}, H^{\dagger} Q d^{c} ; \\
c_{2}=\frac{1}{2}: H^{\prime} Q u^{c}, H^{\prime \dagger} L l^{c}, H^{\dagger} Q d^{c}
\end{gathered}
$$




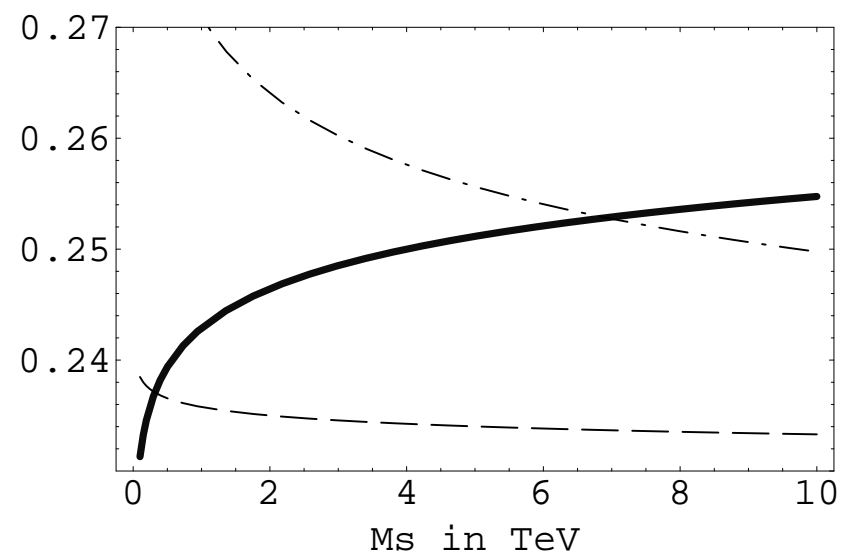

Figure 7: The experimental value of $\sin ^{2} \theta_{W}$ (thick curve), and the theoretical predictions.

Thus, two Higgs doublets are in each case necessary and sufficient to give masses to all quarks and leptons. The presence of the second Higgs doublet changes very little the curves of Fig. 7 and consequently our previous conclusions about $M_{s}$. Two important comments are in order:

(i) The spectrum we assumed in Eq. (3.1) does not contain right-handed neutrinos on the branes. They could in principle arise from open strings in the bulk. Their interactions with the particles on the branes would then be suppressed by the large volume of the transverse space. More specifically, conservation of the three $\mathrm{U}(1)$ charges allow for the following Yukawa couplings involving the right-handed neutrino $\nu_{R}$ :

$$
c_{2}=-\frac{1}{2}: \quad H^{\prime} L \nu_{L} \quad ; \quad c_{2}=\frac{1}{2}: H L \nu_{R}
$$

These couplings lead to Dirac type neutrino masses between $\nu_{L}$ from $L$ and the zero mode of $\nu_{R}$, which is naturally suppressed by the volume of the bulk.

(ii) From Eq. (3.4) and Fig. 7, we find the ratio of the $S U(2)$ and $S U(3)$ gauge couplings at the string scale to be $\alpha_{2} / \alpha_{3} \sim 0.4$. This ratio can be arranged by an appropriate choice of the relevant moduli. For instance, one may choose the color and U(1) branes to be D3 branes while the weak branes to be D7 branes. Then the ratio of couplings above can be explained by choosing the volume of the four compact dimensions of the seven branes to be $V_{4}=2.5$ in string units. This predicts an interesting spectrum of KK states, different from the naive choices that have appeared hitherto: the only SM particles that have KK descendants are the $\mathrm{W}$ bosons as well as the hypercharge gauge boson. However since the hypercharge is a linear combination of the three U(1)'s the massive U(1) gauge bosons do not couple to hypercharge but to doublet number.

\subsection{The fate of $U(1)$ 's, proton stability and neutrinno masses}

The model under discussion has three $U(1)$ gauge interactions corresponding to the gener- 
ators $Q_{1}, Q_{2}, Q_{3}$. From the previous analysis, the hypercharge was shown to be either one of the two linear combinations: $Y=Q_{1} \mp \frac{1}{2} Q_{2}+\frac{2}{3} Q_{3}$. It is easy to see that the remaining two $U(1)$ combinations orthogonal to $Y$ are anomalous. In particular there are mixed anomalies with the SU(2) and SU(3) gauge groups of the Standard Model. These anomalies are canceled by two axions coming from the closed string sector, via the standard GreenSchwarz mechanism [11]. The mixed anomalies with the non-anomalous hypercharge are also canceled by dimension five Chern-Simmons type of interactions [8]. The presence of such interactions has so far escaped attention in the context of string theory.

An important property of the above Green-Schwarz anomaly cancellation mechanism is that the two $U(1)$ gauge bosons $A$ and $A^{\prime}$ acquire masses leaving behind the corresponding global symmetries. This is in contrast to what would had happened in the case of an ordinary Higgs mechanism. These global symmetries remain exact to all orders in type I string perturbation theory around the orientifold vacuum. This follows from the topological nature of Chan-Paton charges in all string amplitudes. On the other hand, one expects non-perturbative violation of global symmetries and consequently exponentially small in the string coupling, as long as the vacuum stays at the orientifold point. Once we move sufficiently far away from it, we expect the violation to become of order unity. So, as long as we stay at the orientifold point, all three charges $Q_{1}, Q_{2}, Q_{3}$ are conserved and since $Q_{3}$ is the baryon number, proton stability is guaranteed.

To break the electroweak symmetry, the Higgs doublets in Eq. (3.5) should acquire non-zero VEV's. Since the model is non-supersymmetric, this may be achieved radiatively [12]. From Eq. (3.6), to generate masses for all quarks and leptons, it is necessary for both higgses to get non-zero VEV's. The baryon number conservation remains intact because both Higgses have vanishing $Q_{3}$. However, the linear combination which does not contain $Q_{3}$, will be broken spontaneously, as follows from their quantum numbers in Eq. (3.5). This leads to an unwanted massless Goldstone boson of the Peccei-Quinn type. The way out is to break this global symmetry explicitly, by moving away from the orientifold point along the direction of the associated modulus so that baryon number remains conserved. Instanton effects in that case will generate the appropriate symmetry breaking couplings in the potential.

A generic feature of the above models is that some of the Standard Model states should correspond to open strings with one end in the bulk, implying the existence of some extra branes, in addition to the ones used above. One can then introduce an extra brane in the bulk with a corresponding $U(1)_{b}$ bulk gauge group. This group is broken by anomalies, leaving behind an additional global symmetry that can be identified with the lepton number [13]. Lepton number conservation is important in particular for the extra dimensional neutrino mass suppression mechanism described above, that can be destabilized by the presence of a large Majorana neutrino mass term. Such a term can be generated by the lepton-number violating dimension five effective operator $L L H H$ that leads, in the case of $\mathrm{TeV}$ string scale models, to a Majorana mass of the order of a few $\mathrm{GeV}$. Even if we manage to eliminate this operator in some particular model, higher order operators would also give unacceptably large contributions, as we focus on models in which the ratio between the Higgs vacuum expectation value and the string scale is just of order 


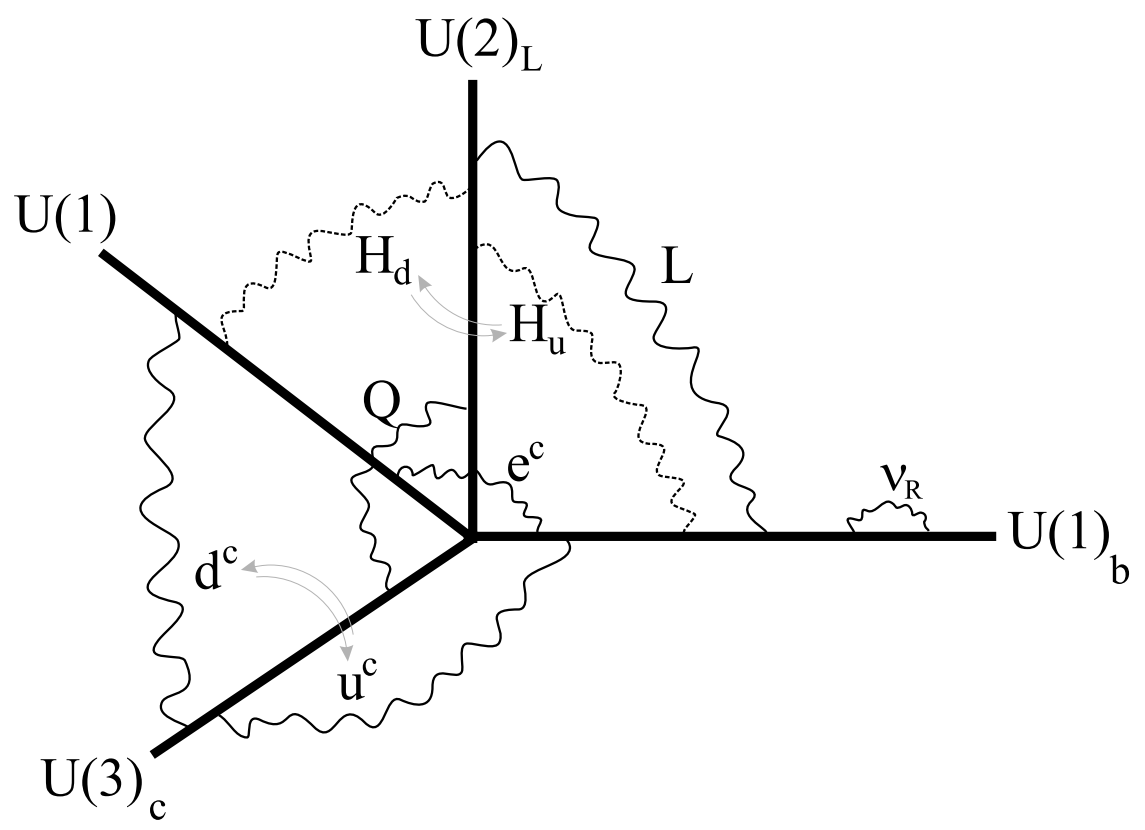

Figure 8: Pictorial representation of the D-brane models presented above. The $U(3)_{c}$ color, $U(2)_{L}$ weak and $U(1)_{b}$ bulk branes point in three orthogonal (complex) directions of the six-dimensional internal space. On the other hand, the $U(1)$ brane is on top of the color or the weak branes, leading to a prediction for $\sin ^{2} \theta_{W}$ analyzed above.

$\mathcal{O}(1 / 10)$. The best way to protect tiny neutrino masses from such contributions is to impose lepton number conservation. The corresponding D-brane models are shown pictorially in Fig. 8.

\section{Conclusions}

Clearly, today, these theories exist only in our imagination. However, we look forward at the next generation of high energy experiments and in particular at the most powerful machine, the LHC at CERN. In fact, it is designed since last decade to explore the origin of mass of elementary particles and to test, in particular, the idea of supersymmetry, looking for the production of superparticles. We now hope that this accelerator may discover more spectacular and "exotic" phenomena, such as the existence of large extra dimensions of space and of fundamental strings.

\section{Acknowledgments}

This work was partly supported by the European Commission under RTN contract HPRNCT-2000-00148.

\section{References}

[1] I. Antoniadis, Phys. Lett. B 246 (1990) 377. 
[2] N. Arkani-Hamed, S. Dimopoulos and G. Dvali, Phys. Lett. B 429 (1998) 263; I. Antoniadis, N. Arkani-Hamed, S. Dimopoulos and G. Dvali, Phys. Lett. B 436 (1998) 263.

[3] C.D. Hoyle, U. Schmidt, B.R. Heckel, E.G. Adelberger, J.H. Gundlach, D.J. Kapner and H.E. Swanson, Phys. Rev. Lett. 86 (2001) 1418; J. Chiaverini, S. J. Smullin, A. A. Geraci, D. M. Weld and A. Kapitulnik, hep-ph/0209325; J. C. Long, H. W. Chan, A. B. Churnside, E. A. Gulbis, M. C. Varney and J. C. Price, hep-ph/0210004; D. E. Krause, E. Fischbach, Lect. Notes Phys. 562,292 (2001) [hep-ph/9912276]; H. Abele, S. Haeßler and A. Westphal, in 271th WE-Heraeus-Seminar, Bad Honnef, 25.2.-1.3.2002.

[4] I. Antoniadis and K. Benakli, Phys. Lett. B 326 (1994) 69; I. Antoniadis, K. Benakli and M. Quirós, Phys. Lett. B 331 (1994) 313 and Phys. Lett. B 460 (1999) 176; P. Nath, Y. Yamada and M. Yamaguchi, Phys. Lett. B 466 (1999) 100 T.G. Rizzo and J.D. Wells, Phys. Rev. D 61 (2000) 016007; T.G. Rizzo, hep-ph/9909232; A. De Rujula, A. Donini, M.B. Gavela and S. Rigolin, Phys. Lett. B 482 (2000) 195; E. Accomando, I. Antoniadis and K. Benakli, Nucl. Phys. B 579 (2000) 3.

[5] G.F. Giudice, R. Rattazzi and J.D. Wells, Nucl. Phys. B 544 (1999) 3; E.A. Mirabelli, M. Perelstein and M.E. Peskin, Phys. Rev. Lett. 82 (1999) 2236; T. Han, J. D. Lykken and R. Zhang, Phys. Rev. D 59 (1999) 105006; K. Cheung and W.-Y. Keung, Phys. Rev. D 60 (1999) 112003; C. Balázs et al., Phys. Rev. Lett. 83 (1999) 2112; L3 Collaboration (M. Acciarri et al.), Phys. Lett. B 464 (1999) 135, Phys. Lett. B 470 (1999) 281; J.L. Hewett, Phys. Rev. Lett. 82 (1999) 4765; D. Atwood, C.P. Burgess, E. Filotas, F. Leblond, D. London and I. Maksymyk, hep-ph/0007178.

[6] K.R. Dienes, E. Dudas and T. Gherghetta, Phys. Lett. B 436 (1998) 55; Nucl. Phys. B 537 (1999) 47.

[7] C. Bachas, JHEP 9811, 23 (1998); I. Antoniadis and C. Bachas, Phys. Lett. B 450 (1999) 83; N. Arkani-Hamed, S. Dimopoulos and J. March-Russell, hep-th/9908146; Antoniadis, C. Bachas and E. Dudas, Nucl. Phys. B 560 (1999) 93.

[8] I. Antoniadis, E. Kiritsis and T. Tomaras, Phys. Lett. B 486 (2000) 186.

[9] G. Shiu and S.-H.H. Tye, Phys. Rev. D 58 (1998) 106007, hep-th/9805157; Z. Kakushadze and S.-H.H. Tye, Nucl. Phys. B 548 (1999) 180, hep-th/9809147; L.E. Ibáñez, C. Muñoz and S. Rigolin, hep-ph/9812397.

[10] N. D. Lambert and P. C. West, JHEP 9909, 021 (1999); G. Aldazabal, L.E. Ibáñez and F. Quevedo, hep-th/9909172 and hep-ph/0001083.

[11] A. Sagnotti, Phys. Lett. B 294 (1992) 196; L.E. Ibáñez, R. Rabadán and A.M. Uranga, Nucl. Phys. B 542 (1999) 112; E. Poppitz, Nucl. Phys. B 542 (1999) 31.

[12] I. Antoniadis, K. Benakli and M. Quirós, Nucl. Phys. B 583 (2000) 35.

[13] I. Antoniadis, E. Kiritsis, J. Rizos and T. N. Tomaras, Nucl. Phys. B 660 (2003) 81. 